Joanna Bielińska ${ }^{1}$, Ariel Liebert ${ }^{1}$, Natalia Lesiewska², Maciej Bieliński², Artur Mieczkowski², Paulina Sopońska-Brzoszczyk², Bartosz Brzoszczyk², Dominika Długosz², Wojciech Guenter², Alina Borkowska², Maria Kłopocka

\title{
Depressive and anxiety symptoms among patients with inflammatory bowel diseases
}

\author{
Corresponding author: \\ Joanna Bielińska, MD \\ Chair of Vascular \\ and Internal Diseases \\ Collegium Medicum of Nicolaus \\ Copernicus University \\ 75 Ujejskiego St. \\ 85-168 Bydgoszcz, Poland \\ E-mail: bielinskaj@gmail.com
}

Medical Research Journal 2017:

Volume 2, Number 1, 6-12

10.5603/MRJ.2017.0002

Copyright (C) 2017 Via Medica ISSN 2451-2591

\begin{abstract}
Introduction. This study was conducted on a population of patients with inflammatory bowel disease (IBD) and was based on an assessment of the prevalence and severity of depression and anxiety symptoms in various clinical aspects. The psychological features of IBD patients are very important in the perception of symptoms, but crucial as triggers of IBD or as a releasing factor for IBD symptoms recurrence.

Methods. The study included 130 patients with IBD, including 68 with Crohn's disease (CD) and 62 with ulcerative colitis (UC). The severity of anxiety and depression symptoms were examined by the Hospital Anxiety and Depression Scale (HADS). Patients were also subjected to assessment of anthropometric attributes, clinical factors, quality of life, and symptoms of the disease, with dedicated clinical scales. Results. The occurrence of significant symptoms of anxiety was estimated at $45.5 \%$ in patients with CD and $30.5 \%$ in the UC patients. Significant depressive symptoms related to $20.5 \%$ of people with CD and $17.5 \%$ of patients with UC. The parameters of anxiety and depression showed significant associations with parameters of quality of life, BMI, and the scales describing the exacerbation of the diseases.

Conclusions. The analyses did not reveal significant differences in the severity and prevalence of symptoms of anxiety and depression in subgroups with IBD. The expansion of relevant symptoms of anxiety and depression in this population was greater than in the general population. In addition, there was a significant correlation between parameters of HADS and clinical factors.

Key words: depression, anxiety, inflammatory bowel disease, Crohn's disease, ulcerative colitis
\end{abstract}

Med Res J 2017; 2 (1): 6-12

\section{Introduction}

The prevalence of the inflammatory bowel diseases (IBD) in adult Europeans is high; it reaches nearly $140 / 100,000$ people [1]. It is a group of chronic and recurrent diseases, comprised of Crohn's disease (CD) and ulcerative colitis (CU). Both of these conditions are characterised by the occurrence of phases, with periods of remission and relapse. Genetic, environmental, immunological, and psychosocial factors have an impact on the course and progression of IBD [2, 3]. The symptoms of IBD, as well as their complications (which are often numerous and serious), impair the lives of the patients in a severe way, lowering their quality of life [4-6]. The abovementioned is associated with the occurrence of depressive symptoms and anxiety in this group of patients [7].
Crohn's disease can have clinical onset at any age; however, it starts mostly in the second or third decade of life. In $25 \%$ of cases the diagnosis is made before the age of 18 years [8]. The estimated prevalence of $C D$ is 12.7 cases per 100,000 people in Europe [9], and it has similar prevalence in women and men [1]. The disease can affect any part of the gastrointestinal tract, from the oral cavity to the anus. It is first located in the mucosa, and it spreads to the rest of the intestinal wall afterwards, which may cause many complications (narrowings, fistulas, abscesses). Affected segments are separated by healthy fragments of the gastrointestinal tract.

Ulcerative colitis is more frequent in Europe than CD (24.3 cases per 100,000 people) [9]. The disease may occur at any age; there are two peaks in incidence rates: between the age 15 and 30 years as well as (smaller) 50 and 70 years $[10,11]$. Unlike CD, it affects only the 
mucosa and is restricted to the large intestine; it spreads continuously from the anus in the proximal direction.

Depression is the disease most often co-existing with IBD [12]. The higher prevalence of depressive symptoms in the group of $\mathrm{CU}$ patients was shown (24\%) as opposed to CD patients (15\%) [7]. Relevant differences were found in the prevalence and severity of depressive symptoms depending on the phase of the disease [13]. In patients without any earlier psychological disorders, the risk factors of developing depression in the future were: female sex, aggressive course of the disease, and advanced endoscopic activity [14]. In the population of IBD patients who were over 65 years old, the risk factors were: lower education, treatment with corticosteroids, and lower physical activity; depression was associated with worse cooperation in treatment [15]. The sustainability of IBD symptoms despite treatment was likewise a risk factor for development of depression [12].

In $C D$, the risk of depression is higher when there is intense activity of the disease and low socioeconomic status. Older age and experiencing stress were observed to be correlated with depression [16]. During a 5-year observation of over a thousand patients after a surgical procedure (707 patients with $C D$ and 530 with $\mathrm{CU})$ it was found that in the case of CD there is higher (around 16\%) risk of depression than in CU. In CD, risk factors were: female sex, surgery in the first 3 years from the diagnosis, other concomitant diseases, immunosuppressive drugs, ostomy and active inflammatory process in the anal area [17]. The risk of depression was $44 \%$ when 4 of these factors were present.

The factors connected with $\mathrm{CU}$ which in the studies showed correlation with depressive symptoms were: experiencing stress, activity of the disease, and hospitalisations [16]. Surgery in CU caused $11 \%$ risk of developing depressive symptoms in five years. Other significant factors were female sex and other co-existing diseases [17].

The anxiety symptoms among patients with IBD are two times more frequent than in the general population [18]. In this group, the factors that correlated most with anxiety were: chronic pain, female sex, and childhood sexual abuse [18]. Wider prevalence was observed during clinical exacerbation of IBD (80\%) in comparison to remission (29\%) [19]. It is thought that the main factors responsible for anxiety symptoms in the IBD population are: the awareness of incurability, the fear of surgical intervention, and the fear of neoplasm development [20]. It was likewise observed that religious patients experience lesser intensity of anxiety symptoms.

In regard to Crohn's disease, Goodhand et al. found that experiencing stress, abdominal pain, and low socioeconomic status have an influence on the advancement of anxiety symptoms [16].
In the course of ulcerative colitis, the anxiety symptoms were more frequent in groups of patients suffering from stress and in those who were recently diagnosed [16].

As mentioned, there is clear evidence that in IBD the anxiety and depressive symptoms occur frequently. Their prevalence has been reported to be as high as $80 \%$ and $60 \%$, respectively, during relapses [21] and in up to $35 \%$ of patients in the remission state [22, 23]. The anxiety and depressive symptoms are likewise the main psychological factors influencing the clinical course of IBD [24]. Hence, the aim of this study was to evaluate the appearance and progression of anxiety and depressive symptoms within individuals with different activity of the disease.

\section{Materials and methods}

The study involved 130 patients: 68 with CD ( 29 women and 38 men) and 62 with CU (29 women and 32 men). They were of Polish nationality and Caucasian ethnicity. Patients were treated on an outpatient basis. During the observation, the diagnosis did not change. Demographic factors are shown in Table 1.

Permission for the study was obtained from the Bioethical Commission of Nicolaus Copernicus University, Collegium Medicum in Bydgoszcz. The subjects demonstrated their willingness to participate in the project by signing an Informed Consent Form. All the patients were informed about the goal and process of the study, potential risks, anonymity of the data, and the possibility of cancelling their participation at any moment.

\section{Assessments}

The procedure of examination shown below was conducted once and consisted of clinical, biochemical, and psychological assessment.

Clinical assessment consisted of obtaining a medical history, performing a physical examination, and filling out a questionnaire concerning the course of the disease and individual factors.

The following types of parameters were assessed:

- demographic (age, sex, education, place of residence, etc.);

- relating to illness (disease type, duration, other diseases, risk factors of IBD or exacerbations, anthropometric parameters, etc.).

Anthropometric assessment: weight, height, and BMI (Body Mass Index) were obtained.

Scales describing the activity and course of the disease - scales used in everyday practice for monitoring patients were used:

- for CD patients:

- CDAl (Crohn's Disease Activity Index), 
Table 1. Basic demographic and clinical parameters in subgroups of patients with Crohn's disease (CD) and ulcerative colitis (CU)

\begin{tabular}{|c|c|c|c|}
\hline Parameter & $C D(n=68)$ & $C U(n=62)$ & $\mathbf{p}$ \\
\hline Age (y) & $28.5(22.0-36.5)$ & $31.0(21.0-50.0)$ & 0.41 \\
\hline Gender (n, \%) & $\begin{array}{l}\text { o } 30(44 \%) \\
\text { > } 38 \text { (56\%) }\end{array}$ & $\begin{array}{l}\text { o } 31 \text { (50\%) } \\
\text { o } 31 \text { (50\%) }\end{array}$ & 0.63 \\
\hline Duration of illness $(\mathrm{y})$ & $5.0(3.0-8.0)$ & $6.0(2.0-8.0)$ & 0.74 \\
\hline \multicolumn{4}{|l|}{ Education } \\
\hline Basic (n, \%) & $4(6 \%)$ & $4(6.5 \%)$ & 0.82 \\
\hline Vocational (n, \%) & $11(16 \%)$ & $8(13 \%)$ & \\
\hline Secondary (n, \%) & 33 (48.5\%) & $31(50 \%)$ & \\
\hline Higher (n, \%) & 20 (29.5\%) & 19 (30.5\%) & \\
\hline \multicolumn{4}{|l|}{ Physical activity } \\
\hline None (n, \%) & $25(37 \%)$ & 24 ( $39 \%)$ & 0.60 \\
\hline$<1 /$ week & $8(12 \%)$ & $14(22.5 \%)$ & \\
\hline 2-3/week & 31 (45.5\%) & $16(26 \%)$ & \\
\hline$>3 /$ week & $4(5.5 \%)$ & $8(12.5 \%)$ & \\
\hline BMI $\left[\mathrm{kg} / \mathrm{m}^{2}\right]$ & $22.1(19.2-24.8)$ & $22.5(20.2-26.3)$ & 0.19 \\
\hline Waist $[\mathrm{cm}]$ & $81.0(76.0-89.0)$ & $83.0(76.0-90.0)$ & 0.55 \\
\hline Nicotinism (n. \%) & $11(16 \%)$ & $14(23 \%)$ & 0.81 \\
\hline
\end{tabular}

Data is presented as median (Q25-Q75) or number and percentage. BMI - body mass index

- for CU patients:

- Mayo Classification (The Mayo Score).

Quality of life was examined with the dedicated questionnaire - IBDQ (Inflammatory Bowel Disease Questionnaire). The IBDQ was used under license of McMaster University (Order \# 29620 and Order \#29621).

Psychological assessment applied mostly to the details of intensification of the depressive symptoms and anxiety, which were assessed using the HADS scale (Hospital Anxiety and Depression Scale).

\section{Statistical analysis}

STATISTICA 12.5 was used for statistical analyses; it was conducted for two groups.

The distribution of variables was analysed using the Shapiro-Wilk test. To assess the significance of the differences between groups, the Mann-Whitney $U$ test was used. The R-Spearman test was used to determine correlations between non-parametrical variables. Significant differences were assumed for $p<0.05$.

\section{Results}

\section{Subjects and baseline characteristics}

Of the 130 patients who were invited to participate the study, 68 had a diagnosis of CD and 62 had a diagnosis of CU. There were no significant differences
Table 2. HADS results in the study group

\begin{tabular}{lccc}
\hline & CD $(\mathbf{n}=\mathbf{6 8})$ & $\mathbf{C U}(\mathbf{n}=\mathbf{6 2})$ & $\mathbf{p}$ \\
\hline HADS_A & $7.0(5.0-9.0)$ & $6.0(4.0-9.0)$ & 0.69 \\
HADS_D & $4.0(2.0-7.0)$ & $4.0(2.0-7.0)$ & 0.90 \\
& Female $(\mathbf{n}=59)$ & Male $(\mathbf{n}=\mathbf{7 1})$ & $\mathbf{p}$ \\
HADS_A & $8.0(5.0-10.0)$ & $6.0(4.0-8.0)$ & $\mathbf{0 . 0 0 8}$ \\
HADS_D & $4.0(2.0-7.0)$ & $3.0(2.0-7.0)$ & 0.76 \\
\hline
\end{tabular}

Data presented as median (Q25-Q75). CD - Crohn's disease; CU - ulcerative colitis; HADS_A - Hospital Anxiety and Depression Scale, subscale of anxiety; HADS_D - Hospital Anxiety and Depression Scale, subscale of depression

in the demographic and clinical parameters between the $C D$ and $C U$ groups with regards to age, gender, duration of illness, education, physical activity, BMI, waist circumference, and nicotinism (Tab. 1).

\section{Anxiety and depressive symptoms \\ in the studied population}

In the whole group of IBD patients the median value for severity of anxiety symptoms reached 7.0 and the median value for severity of depression was 4.0. There was no difference between the $C D$ and $C U$ groups in HADS_D scores, while the CD group gained insignificantly higher HADS_A results (Tab. 2). However, significantly higher results of HADS_A scale were observed in the group of women versus men $(p=0.008)(T a b .2)$. The analysis of the correlations between HADS_A and 
Table 3. Spearman rank correlations of Hospital Anxiety and Depression Scale (HADS) subscales results with clinical parameters

\begin{tabular}{lcccc}
\hline Parameter & HADS_A & P & HADS_D & P \\
\hline Age $(\mathrm{y})$ & 0.137002 & 0.14 & 0.294311 & $\mathbf{0 . 0 0 0 7}$ \\
Duration of illness $(\mathrm{y})$ & 0.045748 & 0.65 & -0.059304 & 0.57 \\
BMl & -0.080805 & 0.36 & 0.211421 & $\mathbf{0 . 0 1 6}$ \\
\hline
\end{tabular}

HADS A - Hospital Anxiety and Depression Scale, subscale of anxiety; HADS D — Hospital Anxiety and Depression Scale, subscale of depression; $\overline{B M I}$ - body mass index

Table 4. Results of Hospital Anxiety and Depression Scale (HADS) in subgroups of patients with Crohn's disease (CD) and ulcerative colitis (CU) in remission and exacerbation

\begin{tabular}{lccc}
\hline & Remission & Exacerbation & p \\
\cline { 2 - 3 } & $\mathbf{C D}(\mathbf{n}=\mathbf{1 7})$ & $\mathbf{C D}(\mathbf{n}=\mathbf{5 1})$ & \\
\hline HADS_A & $5.0(0.0-8.0)$ & $8.0(1.0-19.0)$ & $\mathbf{0 . 0 0 1}$ \\
HADS_D & $2.0(0.0-9.0)$ & $5.0(0.0-16.0)$ & 0.07 \\
& $\mathbf{C U}(\mathbf{n}=\mathbf{2 0})$ & $\mathbf{C U}(\mathbf{n}=\mathbf{4 2})$ & $\mathbf{p}$ \\
HADS_A & $2.0(0.0-8.0)$ & $5.0(0.0-13.0)$ & 0.5 \\
HADS_D & $5.0(0.0-12.0)$ & $6.0(0.0-16.0)$ & $\mathbf{0 . 0 1 9}$ \\
\hline
\end{tabular}

Data presented as median (Q25-Q75) exacerbation and those who met criteria for remission of the disease symptoms. In patients with $C D$, subjects with exacerbation had a significantly higher levels of anxiety symptoms $(p=0.001)$, while CU patients with exacerbation were characterised by significantly higher levels of depressive symptoms $(p=0.019)($ Tab. 4).

\section{Anxiety symptoms and quality of life}

Fifty patients presented clinically significant anxiety symptoms (38\% of the study group). There were no significant differences between this group and the other patients in terms of age, disease duration, and BMI. However, all IBDQ parameters were significantly worse in this group (Tab. 5).

Table 5. The Inflammatory Bowel Disease Questionnaire (IBDQ) results and clinical parameters in groups with and without clinically significant symptoms of anxiety

\begin{tabular}{lccc}
\hline Parameter & HADS_A $>\mathbf{7}(\mathbf{n}=\mathbf{5 0})$ & HADS_A $\leq \mathbf{7}(\mathbf{n}=\mathbf{8 0})$ & $\mathbf{p}$ \\
\hline Age $(\mathrm{y})$ & $31.0(22.0-46.0)$ & $27.0(20.0-39.0)$ & 0.30 \\
Gender & $+28(56 \%)$ & $+31(39 \%)$ & $\mathbf{0 . 0 1 8}$ \\
Duration of illness $(\mathrm{y})$ & $22(44 \%)$ & $5.0(2.0-8.0)$ & 0.82 \\
BMI & $5.0(3.0-8.0)$ & $22.5(20.4-25.8)$ & 0.14 \\
IBDQ (total) & $21.3(18.9-24.1)$ & $173(151-200)$ & $\mathbf{0 . 0 0 0 2}$ \\
IBDQ emotional function & $154.5(131-177)$ & $55(46.5-62.0)$ & $\mathbf{0 . 0 0 2}$ \\
IBDQ bowel function & $47(39-54)$ & $28(24-32)$ & $\mathbf{0 . 0 0 0 4}$ \\
IBDQ social function & $23.5(17.5-28)$ & $66(57.5-76.5)$ & $\mathbf{0 . 0 0 0 9}$ \\
IBDQ systemic function & $57.5(48.5-68)$ & $28(24-31)$ & $\mathbf{0 . 0 0 0 0 9}$
\end{tabular}

Data presented as median (Q25-Q75) or number and percentage. BMI — body mass index; HADS_A — Hospital Anxiety and Depression Scale, subscale of anxiety

HADS_D results with clinical parameters revealed statistically significant positive association of depression subscale with age $(r=0.29 ; p=0.0007)$ and $B M I$ $(r=0.21 ; p=0.01)$ (Tab. 3). Also, the group of patients with overweight achieved significantly higher median levels of depressive symptoms (6.0 vs. 3.5, $p=0.04$ ).

The clinical CDAl scale for CD and Mayo for CU was used to determine the patients who met the criteria for

\section{Depressive symptoms and quality of life}

A similar analysis was also performed for depressive symptoms. It was found that significant depressive symptoms occurred in 30 persons (23\% of the study group). These patients did not differ from others in terms of gender and duration of illness. There was a difference in measure of tendency of BMI (24.1 vs. 21.8; $p=0.09$ ). 
Table 6. The Inflammatory Bowel Disease Questionnaire (IBDQ) results and clinical parameters in groups with and without clinically significant symptoms of depression

\begin{tabular}{lccc}
\hline Parameter & HADS_D $>\mathbf{7}(\mathbf{n}=\mathbf{3 0})$ & HADS_D $\leq \mathbf{7}(\mathbf{n}=\mathbf{1 0 0})$ & $\mathbf{p}$ \\
\hline Age $(\mathrm{y})$ & $39.0(31.0-51.0)$ & $26.0(20.0-37.0)$ & $\mathbf{0 . 0 0 0 1}$ \\
Gender & $+15(50 \%)$ & $+44(44 \%)$ & 0.75 \\
Duration of the illness $(\mathrm{y})$ & $015(50 \%)$ & $556(56 \%)$ & 0.33 \\
BMI & $6.0(3.0-10.0)$ & $5.0(2.5-7.0)$ & 0.09 \\
IBDQ (total) & $24.1(20.3-27.6)$ & $21.8(19.2-24.4)$ & $\mathbf{0 . 0 0 0 5}$ \\
IBDQ emotional function & $147(132-163)$ & $172(146-199)$ & $\mathbf{0 . 0 0 3}$ \\
IBDQ bowel function & $47(39-52)$ & $53(44-61)$ & $\mathbf{0 . 0 0 0 3}$ \\
IBDQ social function & $23(18-25)$ & $28(23-32)$ & $\mathbf{0 . 0 0 0 4}$ \\
IBDQ systemic function & $57(49-63)$ & $66(57-76)$ & $\mathbf{0 . 0 0 0 2}$ \\
\hline
\end{tabular}

Data presented as median (Q25-Q75) or number and percentage. BMI — body mass index; HADS_D — Hospital Anxiety and Depression Scale, subscale of depression

As with anxiety symptoms, all aspects of quality of life measured by IBDQ were significantly lower in the group with HADS_D-positive results.

\section{Discussion}

For many years clinicians and scientists have observed and described the psychological aspects in the group of patients who suffer from IBD. Relations between etiopathogenesis, clinical aspects of IBD, and psychological factors are still not well understood, despite the utilisation of more and more exact scientific methods. Such facets are very important considering the development of the disease and its course. In this study, we analysed the correlation between demographic, clinical, and biochemical factors and the occurrence and the intensity of depressive and anxiety symptoms.

Depressive and anxiety disorders are more frequent in patients diagnosed with IBD than in the main population [12, 18]. The literature shows evidence of significant association between the activity of the disease and the occurrence of depressive symptoms and anxiety. Although depressive symptoms are more frequent in patients with $\mathrm{CU}$, the anxiety disorders are slightly more common in individuals with CD. The data obtained in this study also confirm the existence of positive correlations between disease activity and symptoms of anxiety and depression.

In comparison to the literature, the prevalence of depressive symptoms was smaller in the experimental group. Various publications describe patients in remission, whose depressive symptoms reach approximately $35 \%[22,23]$. In patients with exacerbation, significant depressive symptoms concern $60 \%$ of patients [21].
Practically all papers describing the relationship between the clinical course of IBD and depressive and anxiety symptoms show greater intensity during the course of the disease activity. A meta-analysis of 30 studies from 1994 to 2014 indicates a significant correlation between the severity of IBD symptoms and the variety of psychological symptoms [25]. It is very important that there are significant differences in the prevalence and severity of depressive disorders depending on the disease phase [19]. Thus, in the course of exacerbation, the prevalence of significant depressive symptoms was $60 \%$ [21]. By contrast, in the remission phase this was approximately $35 \%[22,23]$.

The literature is dominated by publications in which excessive body weight is associated with a tendency to exacerbate depressive and anxiety symptoms. The cause of this phenomenon is seen in the inflammatory system that is modulated by obesity [26]. It was found that in a group of 10,348 women, BMI was positively correlated with mild to moderate depressive disorders and also with high levels of depression. It has also been reported that the degree of obesity was an independent risk factor for the occurrence of depressive disorders also in the population of obese women. Abdominal obesity independently correlated positively with depressive symptoms [27]. Population studies in Canada have shown that in obese people mood disorders occurred two times more frequently than in healthy populations and $30 \%$ more often than in overweight populations [28].

In recent epidemiological studies, the incidence of obesity or overweight among IBD patients is estimated at $15-40 \%$, and their occurrence significantly complicates treatment, particularly the effectiveness of surgical intervention [29]. It is believed that IBD and obesity have many common elements, including intestinal microbiota, diet, and proinflammatory features of intra-abdominal adipose tissue [30]. 
In this study, the incidence of obesity was not associated with a significantly higher level of anxiety, whereas patients with obesity were significantly more depressed. To conclude, individuals with a clinically significant burden of malnutrition do not have higher levels of anxiety and depression.

The literature includes observations describing a decrease in quality of life in IBD patients, which is significantly greater in the CD subgroup [31]. There are also studies that show no differences in the quality of life in subgroups with different IBD [32]. Such discrepancies may result from complications more commonly appearing in CD patients, for instance intestinal and parenteral disease [31]. Likewise, the specific quality of life assessment method utilised in particular studies might be responsible for inconsistent findings.

In our study, the occurrence of significant depressive and anxiety symptoms correlated with poorer IBDQ scores. During their daily living, IBD patients have to face a variety of issues and challenges related to heightened levels of psychological distress. It includes low self-esteem, the feeling of being stigmatised, or disturbances in employment and relationships [25]. Likewise, the deterioration of the disease, which results from the elevated inflammatory response, related to both anxiety and depression, may account for the poorer outcome of quality of life questionnaires [26].

\section{Conclusions}

This study showed a high prevalence of depression and anxiety in the group of patients with IBD. According to our results, one fifth of patients with $C D$ and one in six with $\mathrm{CU}$ presented depressive symptoms. Nearly half of CD patients and one third of individuals with $\mathrm{CU}$ demonstrated significant anxiety symptoms. In regard to gender, women manifested anxiety symptoms more often than men.

Our study also showed that higher severity of depressive and anxiety symptoms is associated with greater clinical disease activity and worse quality of life scores. Moreover, the intensity of depressive symptoms was increased in the individuals who presented higher $\mathrm{BMI}$ values and hence were more obese.

This study proves that psychiatric disorders are very common in IBD patients and have a detrimental impact in the course of the disease. Therefore, it is considerable to take mental health into consideration when making the decisions about planning treatment in patients who suffer from IBD.

\section{Acknowledgements}

This research was made using funds for the statutory activities of Collegium Medicum's (NCU) scientific units.

\section{Conflict of interest: none declared.}

\section{References}

1. Lucendo AJ, Hervías D, Roncero Ó, et al. Epidemiology and temporal trends (2000-2012) of inflammatory bowel disease in adult patients in a central region of Spain. Eur J Gastroenterol Hepatol. 2014; 26(12): 1399-1407, doi: 10.1097/MEG.0000000000000226, indexed in Pubmed: 25341061

2. Elson CO, Cong Y, McCracken VJ, et al. Experimental models of inflammatory bowel disease reveal innate, adaptive, and regulatory mechanisms of host dialogue with the microbiota. Immunol Rev. 2005; 206: 260-276, doi: 10.1111/j.0105-2896.2005.00291.x, indexed in Pubmed: 16048554.

3. Zhang YZ, Li YY. Inflammatory bowel disease: pathogenesis. World J Gastroenterol. 2014; 20(1): 91-99, doi: 10.3748/wjg.v20.i1.91, indexed in Pubmed: 24415861

4. Zhiqin W, Palaniappan S, Raja Ali RA. Inflammatory Bowel Disease-related Colorectal Cancer in the Asia-Pacific Region: Past, Present, and Future. Intest Res. 2014; 12(3): 194-204, doi: 10.5217/ir.2014.12.3.194, indexed in Pubmed: 25349593.

5. Lix LM, Graff LA, Walker JR, et al. Longitudinal study of quality of life and psychological functioning for active, fluctuating, and inactive disease patterns in inflammatory bowel disease. Inflamm Bowel Dis. 2008; 14(11): 1575-1584, doi: 10.1002/ibd.20511, indexed in Pubmed: 18512245

6. Byeon YuMi, Lee J, Lee SJ, et al. Peritonsillar Involvement in Pyoderma Gangrenosum associated with Ulcerative Colitis. Intest Res. 2014; 12(2): 153-156, doi: 10.5217/ir.2014.12.2.153, indexed in Pubmed: 25349583.

7. Kim MC, Jung YS, Song YS, et al. Factors Associated with Anxiety and Depression in Korean Patients with Inactive Inflammatory Bowel Disease. Gut Liver. 2016; 10(3): 399-405, doi: 10.5009/gnl15188, indexed in Pubmed: 26470768.

8. Laass M, Roggenbuck D, Conrad K. Diagnosis and classification of Crohn's disease. Autoimmunity Reviews. 2014; 13(4-5): 467-471, doi: 10.1016/j.autrev.2014.01.029

9. Molodecky NA, Soon IS, Rabi DM, et al. Increasing incidence and prevalence of the inflammatory bowel diseases with time, based on systematic review. Gastroenterology. 2012; 142(1): 46-54.e42; quiz e30, doi: 10.1053/i.gastro.2011.10.001, indexed in Pubmed: 22001864.

10. Stepaniuk P, Bernstein CN, Targownik LE, et al. Characterization of inflammatory bowel disease in elderly patients: A review of epidemiology, current practices and outcomes of current management strategies. Can J Gastroenterol Hepatol. 2015; 29(6): 327-333, indexed in Pubmed: 26069892

11. Conrad K, Roggenbuck D, Laass MW. Diagnosis and classification of ulcerative colitis. Autoimmun Rev. 2014; 13(4-5): 463-466, doi: 10.1016/j.autrev.2014.01.028, indexed in Pubmed: 24424198.

12. Rusu F, Dumitraşcu DL. Four years Follow-up of Patients with Irritable Bowel Syndrome. Rom J Intern Med. 2015; 53(1): 63-72, doi: 10.1515/rjim-2015-0009, indexed in Pubmed: 26076563.

13. Häuser W, Janke KH, Klump B, et al. Anxiety and depression in patients with inflammatory bowel disease: comparisons with chronic liver disease patients and the general population. Inflamm Bowel Dis. 2011; 17(2): 621-632, doi: 10.1002/ibd.21346, indexed in Pubmed: 20848528

14. Panara AJ, Yarur AJ, Rieders B, et al. The incidence and risk factors for developing depression after being diagnosed with inflammatory bowel disease: a cohort study. Aliment Pharmacol Ther. 2014; 39(8): 802-810, doi: 10.1111/apt.12669, indexed in Pubmed: 24588323

15. Long MD, Kappelman MD, Martin CF, et al. Risk factors for depression in the elderly inflammatory bowel disease population. J Crohns Colitis. 2014; 8(2): 113-119, doi: 10.1016/j.crohns.2013.07.002, indexed in Pubmed: 23932782

16. Goodhand JR, Wahed M, Mawdsley JE, et al. Mood disorders in inflammatory bowel disease: relation to diagnosis, disease activity, perceived stress, and other factors. Inflamm Bowel Dis. 2012; 18(12) 2301-2309, doi: 10.1002/ibd.22916, indexed in Pubmed: 22359369.

17. Ananthakrishnan $A N$, Khalili $H$, Pan An, et al. Association between depressive symptoms and incidence of Crohn's disease and ulcerative colitis: results from the Nurses' Health Study. Clin Gastroenterol Hepatol. 2013; 11(1): 57-62, doi: 10.1016/j.cgh.2012.08.032, indexed in Pubmed: 22944733.

18. Fuller-Thomson E, Lateef R, Sulman J. Robust Association Between Inflammatory Bowel Disease and Generalized Anxiety Disorder: Findings from a Nationally Representative Canadian Study. Inflamm Bowel 
Dis. 2015; 21(10): 2341-2348, doi: 10.1097/MIB.0000000000000518, indexed in Pubmed: 26218145.

19. Häuser W, Janke KH, Klump B, et al. Anxiety and depression in patients with inflammatory bowel disease: comparisons with chronic liver disease patients and the general population. Inflamm Bowe Dis. 2011; 17(2): 621-632, doi: 10.1002/ibd.21346, indexed in Pubmed: 20848528

20. Sajadinejad MS, Asgari K, Molavi H, et al. Psychological issues in inflammatory bowel disease: an overview. Gastroenterol Res Pract. 2012; 2012: 106502, doi: 10.1155/2012/106502, indexed in Pubmed: 22778720

21. Addolorato G, Capristo E, Stefanini GF, et al. Inflammatory bowe disease: a study of the association between anxiety and depression physical morbidity, and nutritional status. Scand J Gastroenterol. 1997 32(10): 1013-1021, indexed in Pubmed: 9361174.

22. Mittermaier C, Dejaco C, Waldhoer T, et al. Impact of depressive mood on relapse in patients with inflammatory bowel disease: a prospective 18-month follow-up study. Psychosom Med. 2004; 66(1): 79-84, indexed in Pubmed: 14747641.

23. Andrews $\mathrm{H}$, Barczak $\mathrm{P}$, Allan RN. Psychiatric illness in patients with inflammatory bowel disease. Gut. 1987; 28(12): 1600-1604, indexed in Pubmed: 3428687

24. Ross SC, Strachan J, Russell RK, et al. Psychosocial functioning and health-related quality of life in paediatric inflammatory bowel disease. J Pediatr Gastroenterol Nutr. 2011; 53(5): 480-488, doi: 10.1097/MPG.0b013e31822f2c32, indexed in Pubmed: 21822149

25. Brooks AJ, Rowse G, Ryder A, et al. Systematic review: psychological morbidity in young people with inflammatory bowel disease - risk factors and impacts. Aliment Pharmacol Ther. 2016; 44(1): 3-15, doi: 10.1111/apt.13645, indexed in Pubmed: 27145394.

26. Pierce GL, Kalil GZ, Ajibewa T, et al. Anxiety independently contributes to elevated inflammation in humans with obesity. Obesity (Silver Spring). 2017; 25(2): 286-289, doi: 10.1002/oby.21698, indexed in Pubmed: 28000423

27. Ma J, Xiao L. Obesity and depression in US women: results from the 2005-2006 National Health and Nutritional Examination Survey. Obesity (Silver Spring). 2010; 18(2): 347-353, doi: 10.1038/oby.2009.213, indexed in Pubmed: 19590500.

28. Gadalla TM. Association of obesity with mood and anxiety disorders in the adult general population. Chronic Dis Can. 2009; 30(1): 29-36, indexed in Pubmed: 20031086

29. Singh S, Dulai PS, Zarrinpar A et al Obesity in IBD: epidemiology pathogenesis, disease course and treatment outcomes. Nat Rev Gastroenterol Hepatol. 2017; 14(2): 110-121, doi: 10.1038/nrgastro.2016.181, ndexed in Pubmed: 27899815

30. Harper JW, Zisman TL. Interaction of obesity and inflammatory bowel disease. World J Gastroenterol. 2016; 22(35): 7868-7881, doi: 10.3748/wjg.v22.i35.7868, indexed in Pubmed: 27672284.

31. Nordin K, Påhlman L, Larsson K et al. Health-related quality of life and psychological distress in a population-based sample of Swedish patients with inflammatory bowel disease. Scand J Gastroenterol. 2002; 37(4): 450-457, indexed in Pubmed: 11989837.

32. Kalafateli M, Triantos $C$, Theocharis $G$, et al. Health-related quality of life in patients with inflammatory bowel disease: a single-center experience. Ann Gastroenterol. 2013; 26(3): 243-248, indexed in Pubmed: 24714279 